\title{
Convenio entre la comisión del Gran Canal Interoceánico de Nicaragua y el Gobierno Terrirorial Rama y Kriol
}

\author{
\% Gobierno de Reconciliación \\ y Unidad Nacional \\ mennoun faumsat El Purblo, Presidente!
}

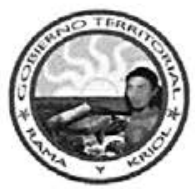

Convenio de Consentimiento Previo, Libre e Informado para la Implementación del Proyecto de Desarrollo del Gran Canal Interoceánico de Nicaragua

\section{Antecedentes:}

1.El 3 de julio de 2012, la Asamblea Nacional de Nicaragua promulga la Ley 800 (Ley del Régimen Jurídico del Gran Canal Interoceánico de Nicaragua y Creación de la Autoridad del Gran Canal Interoceánico de Nicaragua) que declara la construcción del Canal de prioridad e interés supremo nacional.

2. El 14 de junio de 2013, la Asamblea Nacional de Nicaragua otorga a la Empresa HKND (Hong Kong Nicaragua Development Corporation) la concesión para construir el Gran Canal Interoceánico de Nicaragua y crea mediante la Ley 840 (Ley Especial para el Desarrollo de la Infraestructura y Transporte Nicaragüense Atingente al Canal, Zonas de Libre Comercio e Infraestructuras Asociadas) las bases legales para la construcción de la misma.

3.El 7 de julio de 2014, la Empresa Hong Kong Nicaragua Canal Development Investment Company (HKND Group) y el Gobierno de Nicaragua anunciaron la ruta escogida del Canal Interoceánico. La ruta pasa por el territorio ancestral y titulado del Gobierno Territorial Rama y Kriol (GTR-K).

4.El 24 de julio de 2014, se sostuvo una reunión entre el GTR-K y representantes de HKND, Environmental Resources Management (ERM) y la Comisión Nacional de Desarrollo del Gran Canal. El GTR-K expresó sus preocupaciones sobre la Consulta Previa, Libre e Informada en relación al proyecto del Gran Canal, particularmente en lo referido a las disposiciones de la Ley 840 sobre la expropiación de tierras comunales, entre otras y propuso una consulta especial y específica en base a lo establecido en el Convenio número 169 de la OIT, la Ley 28 y la Ley 445.

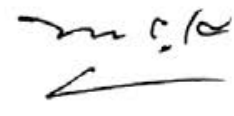


5. En diciembre de 2014, el GTR-K tomó la iniciativa de elaborar lineamientos para que el Gobierno de Nicaragua realice un proceso de consulta en el Territorio Rama y Kriol sobre el proyecto del Gran Canal Interoceánico de Nicaragua.

6. El 4 enero de 2015, se constituye la Comisión del Gobierno de Nicaragua para la realización de la Consulta para obtener el Consentimiento Previo, Libre e Informado para la Implementación del proyecto del Gran Canal Interoceánico de Nicaragua que inicia un diálogo con el GTR-K para construir y aprobar un Documento Armonizado para la implementación del proceso de la Consulta Previa, Libre e Informada que se fundamenta en los lineamientos definidos por el GTR-K.

7.El 27 enero de 2015, el Procurador General de la República, Compañero Hernán Estrada presentó Carta Oficial al Compañero Hector Thomas Mcrea, Presidente del Gobierno Territorial Rama y Kriol que señala:

a. En lo referido al artículo 5, inciso d) y el artículo 12 de la Ley Especial para el Desarrollo de Infraestructura y Transporte Nicaragüense atingente a el Canal, Zonas de Libre Comercio e Infraestructuras Asociadas (Ley 840), manifestamos nuestro compromiso y voluntad de actuar en base a lo establecido en la Ley del Régimen de Propiedad Comunal de los Pueblos Indígenas y Comunidades Étnicas de la Costa Caribe de Nicaragua y los Ríos Bocay, Coco, Indio y Maíz (Ley 445), que establece textualmente en su capítulo V, artículo 24, "El Estado reconoce el Derecho que tienen las Comunidades Indígenas y Étnicas sobre las tierras que tradicionalmente ocupan. De la misma forma reconoce y garantiza la inalienabilidad, inembargabilidad e imprescriptibilidad de las mismas."

b. En relación a la construcción del Gran Canal Interoceánico de Nicaragua e Infraestructuras Asociadas en parte del Territorio Rama y Kriol, la Comisión Nacional de Desarrollo del Gran Canal Interoceánico expresa su disposición de establecer mediante la Consulta Previa, Libre e Informada, un proceso de
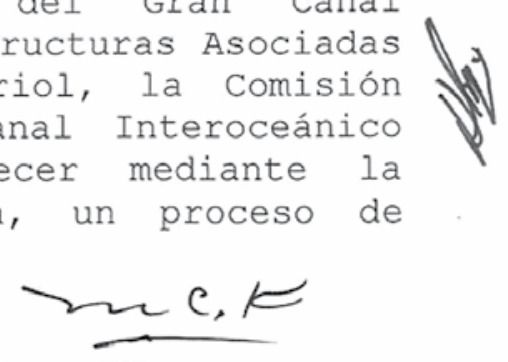
diálogo y negociación que permita definir un canon de arrendamiento para el uso del área geográfica de influencia del proyecto, garantizando de esta manera el legítimo Derecho del Pueblo Rama y Kriol sobre sus tierras y generando bienestar para las generaciones presentes y futuras.

8. Del 4 de febrero al 2 de marzo de 2015, la Comisión del Gobierno de Nicaragua para la realización de la Consulta para obtener el Consentimiento Previo, Libre e Informado para la Implementación del Proyecto del Gran Canal Interoceánico de Nicaragua y el GTR-K, llevaron a cabo la Consulta mediante Asambleas Comunales realizadas en las 9 comunidades que integran el Territorio Rama y Kriol, siendo éstas: Suumu Kaat, Tik Tik Kaanu, Rama Cay, Wiring Cay, Graytown, Indian River, Corn River, Bang Kukuk Taik y Monkey Point.

9.El 28, 29, 30 y 31 de Diciembre, 2015 se celebraron Sesiones de Trabajo de la Comisión del GRUN y GTR-K para elaborar un borrador del Convenio de Consentimiento Previo, Libre e Informado para la Implementación del Proyecto del Gran Canal Interoceánico de Nicaragua a ser sometido ante la Asamblea Territorial para su debida revisión y aprobación.

10. El 8 y 9 de enero, 2016 se celebró la Sesión del GTR-K para revisar el borrador del Convenio de Consentimiento Previo, Libre e Informado para la Implementación del Proyecto del Gran Canal Interoceánico de Nicaragua a ser sometido ante la Asamblea Territorial para su debida revisión y aprobación.

11. El 10 de enero, 2016 se celebró la Asamblea del Territorio Rama y Kriol en la que se autoriza al Gobierno Terriorial Rama y Kriol suscribir el Convenio de Consentimiento Previo, Libre e Informado para la Implementación del Proyecto del Gran Canal Interoceánico de Nicaragua.

Reunidos en Sesión Solemne para la Firma del Convenio de Consentimiento para la Implementación del proyecto de Desarrollo del Gran Canal Interoceánico de Nicaragua, el

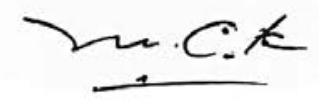


Gobierno Territorial Rama y Kriol (GTR-K) y la Comisión Nacional de Desarrollo del Gran Canal Interoceánico de Nicaragua, señalan:

Considerando:

I

La Comisión Nacional de Desarrollo del Gran Canal de Nicaragua es el órgano de gobierno responsable de la supervisión del uso racional y sostenible de los Recursos Naturales, la protección del ambiente y biodiversidad del área geográfica de influencia alrededor del área de construcción de El Gran Canal Interoceánico de Nicaragua dentro del marco de tratados vinculantes, convenciones internacionales y legislación nacional.

II

El objeto principal de la Comisión será ejercer los derechos y cumplir las obligaciones del Gobierno de la República de Nicaragua en virtud al Acuerdo Marco de Concesión (MCA) y entre sus funciones se contempla: Revisar y aprobar el plan de desarrollo de cada Sub-Proyecto; Suscribir, en nombre y representación de cada Entidad Gubernamental, el Acuerdo de Concesión, así como los Acuerdos de Concesión e Implementación de cada SubProyecto; Emitir todos los Consentimientos que fueren requeridos para el Desarrollo y Operación de El Proyecto y cualquier otro sub-Proyecto y Proponer al Presidente de la República de Nicaragua nuevas leyes 0 reglamentos 0 modificaciones a las leyes y reglamentos que fueren necesarias para el éxito de El proyecto.

\section{III}

El Gobierno Territorial Rama y Kriol es el órgano de administración de la unidad territorial a la cual representa legalmente. El Gobierno Territorial Rama y Kriol, conforme a lo establecido en sus Estatutos y Reglamento Interno ejerce en materia del Derecho al Consentimiento Previo, Libre e Informado, las siguientes funciones:

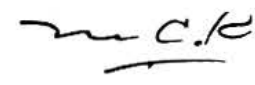


1. Convoca por medio del secretario(a) del GTR-K a los demás miembros a las reuniones ordinarias o extraordinarias y elabora las agendas de las mismas.

2. Decide sobre asumir una relación con terceros, previa consulta y aval de las comunidades, en los casos de permisos de aprovechamiento de tierras en áreas que corresponden a más de una comunidad.

3. Negocia con las autoridades Regionales, Municipales, Departamentales y Central; así como con empresas y otras entidades, los procedimientos y beneficios que obtendrían las comunidades en la implementación de proyectos que podrian afectar el territorio 0 sus comuneros 0 en el aprovechamiento de recursos naturales.

4. Firma convenios, acuerdos y contratos de aprovechamiento con las Autoridades Regionales, Municipales, Central y Empresas Nacionales e Internacionales u otros organismos respecto al uso sostenible de los recursos naturales dentro del territorio.

Es responsabilidad del Gobierno Territorial Rama y Kriol velar por el ejercicio efectivo de los Derechos de los pueblos indígenas y afro descendientes que integran su territorio y fomentar el desarrollo mediante proyectos de inversión que contribuyan al desarrollo económico y social sostenible conforme lo establecido en su Plan Autónomo de Administración y Desarrollo Territorial (PADA), procurando además la protección y preservación del medio ambiente y sus buenas prácticas culturales.

IV

El 18 de diciembre del año 2009 la Comisión Nacional de Demarcación y Titulación (CONADETI) en nombre del Estado de Nicaragua, emitió Título de Pleno Dominio a favor de las 9 comunidades que integran el Territorio Rama y Kriol, habiéndose titulado a su favor 406,849.30 hectáreas de tierra firme de lo que fuera su territorio tradicional, así como 22 cayos integrados al título y 441,308 hectáreas de área marítima otorgadas para el desarrollo de la pesca artesanal.

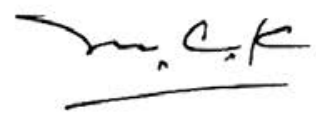


El Territorio Rama y Kriol está integrado por las comunidades Rama de Rama Cay; Wiring Cay; Bang Kukuk Taik (Punta de Águila); Tiktik Kaanu (Zompopera); Suumu Kaat e Indian River (Río Indio); así como las comunidades Kriol de Monkey Point, Corn River (Río Maíz) y Graytown, ubicadas en la Región Autónoma de la Costa Caribe Sur (RACCS) y el Departamento de Río San Juan.

\section{V}

El Gobierno de Nicaragua reconoce que la Consulta Previa, Libre e Informada es un Derecho de los Pueblos Indígenas y Afro descendientes que se sustenta en los artos. 5, 89, 91 y 181 de la Constitución Política de Nicaragua, así como en el Estatuto de Autonomía (Ley 28), el Decreto de la Asamblea Nacional 3584 - Reglamentación del Estatuto de Autonomía y la Ley del Régimen de Propiedad Comunal de los Pueblos Indígenas y Comunidades Étnicas de la Costa Caribe de Nicaragua (Ley 445). En lo referido al Derecho al Consentimiento Previo, Libre e Informado, este proceso se sustenta en el Convenio 169 de la Organización Internacional del Trabajo y la Declaración de Naciones Unidas sobre Derechos de Pueblos Indígenas y Afro descendientes, de las cuales Nicaragua es signataria.

\section{VI}

El proceso de la Consulta para obtener el Consentimiento Previo, Libre e Informado se ha regido por una metodología elaborada en conjunto entre el Gobierno Territorial Rama y Kriol y la Comisión del Gobierno de Nicaragua para la Consulta, que se fundamenta en los principios de Buena Fe, Implementación Previa, Flexibilidad, Transparencia, Exclusividad, Interculturalidad, Información Oportuna, Información Exhaustiva, Confidencialidad, Distribución de los Beneficios, Plazo Razonable e Igualdad.

VII

El proceso de la Consulta se realizó en cada una de las 9 comunidades indígenas y afro descendientes que integran el Territorio Rama y Kriol, contando con una participación de 592 habitantes, quienes reunidos en Asambleas Comunales

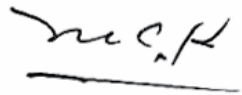


expresaron sus opiniones y preocupaciones acerca del proyecto, así como los beneficios que puede generar.

VIII

A partir de los resultados del proceso de Consulta en las 9 Asambleas Comunales y en Sesión de Trabajo con el Gobierno Territorial Rama y Kriol, se elaboró el primer borrador del presente Convenio de Consentimiento Previo, Libre e Informado para la Implementación del Proyecto del Gran Canal Interoceánico de Nicaragua, mismo que fue sometido a la Asamblea Territorial para su aprobación. En la Asamblea Territorial del Territorio Rama y Kriol, celebrado el 10 de enero, 2016 se presentó dicho borrador del Convenio, mismo que fue aprobado por 54 miembros y que se presenta hoy en ésta Histórica Sesión Solemne para su debida firma, sello e implementación.

Por lo anterior, la Comisión Nacional de Desarrollo del Gran Canal de Nicaragua $y$ el Gobierno Territorial Rama y Kriol, acuerdan lo siguiente:

\section{Clausula Primera: Del Objeto y Legitimidad del Convenio}

1.El objeto del presente Convenio de Consentimiento Previo, Libre e Informado para la Implementación del Proyecto del Gran Canal Interoceánico de Nicaragua es establecer las bases legales para obtener el Consentimiento del Pueblo Indígena Rama y Afro descendiente Kriol para el desarrollo del Canal Interoceánico y Sub-Proyectos en su Territorio, conforme el marco legal nacional e internacional y las normativas del Gobierno Territorial Rama y Kriol que rigen la materia.

2. A través de la firma del presente Convenio de Consentimiento Previo, Libre e Informado, el Gobierno Territorial Rama y Kriol y la Comisión Nacional de Desarrollo del Gran Canal de Nicaragua, aspiran a contribuir al desarrollo humano y el buen vivir del Pueblo Indígena Rama y Afro descendiente Kriol.

3. El presente Convenio cuenta con el Consentimiento del Territorio Rama y Kriol, conforme Actas de Asambleas Comunales, Acta de Sesión del Gobierno Territorial Rama y
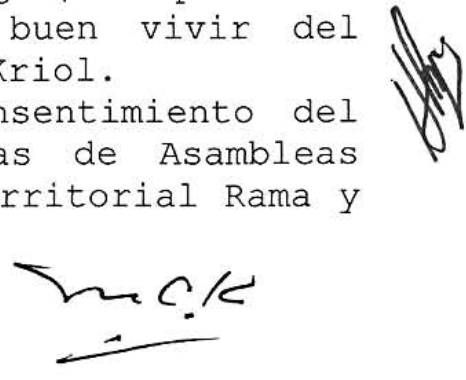
Kriol y Acta de la Asamblea Territorial Rama y Kriol, debidamente firmadas y que se adjuntan.

\section{Clausula Segunda: Del Arriendo del Área Requerida}

4. El Gobierno Territorial Rama y Kriol (GTR-K), en representación del Pueblo Indígena Rama y Afro descendiente Kriol y por mandato expreso de la Asamblea Territorial Rama y Kriol, otorga el requerido Consentimiento Previo, Libre e Informado a la Comisión Nacional de Desarrollo del Gran Canal de Nicaragua para la debida implementación de El proyecto en aproximadamente $263 \mathrm{Km}^{2}$ (Doscientos sesenta y Tres Kilómetros Cuadrados) de su territorio que abarca áreas terrestres y marítimas (Área Requerida).

5. El Área Requerida, siendo propiedad comunal y respetando lo establecido en la Ley del Régimen de Propiedad Comunal de los Pueblos Indígenas y Comunidades Étnicas de la Costa Caribe de Nicaragua y los Ríos Bocay, Coco, Indio y Maíz (Ley 445), que señala en su capítulo V, artículo 24, "El Estado reconoce el Derecho que tienen las Comunidades Indígenas y Étnicas sobre las tierras que tradicionalmente ocupan... y reconoce y garantiza la inalienabilidad, inembargabilidad e imprescriptibilidad de las mismas," permanecerá bajo el pleno dominio de los Pueblos Rama y Kriol.

6. Para el uso del Área Requerida, la Comisión Nacional de Desarrollo del Gran Canal de Nicaragua y el Gobierno Territorial Rama y Kriol suscribirán un contrato de Arrendamiento Indefinido en el que se definirá el canon de arrendamiento anual, pagadero en dólares de los Estados Unidos de América a partir de la firma del Contrato de Arrendamiento.

\section{Clausula Tercera: Del Carácter Vinculante del Convenio de} Consentimiento Previo, Libre e Informado

7. Una vez aprobado, firmado y sellado el presente Convenio de Consentimiento Previo, Libre e Informado para la Implementación del proyecto de Desarrollo del Gran Canal Interoceánico de Nicaragua y Sub Proyectos, este será de estricto y obligatorio cumplimiento para ambas partes.

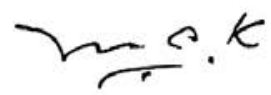


8. Una vez aprobado, firmado y sellado el Presente Convenio de Consentimiento Previo, Libre e Informado para la Implementación del proyecto del Gran Canal Interoceánico de Nicaragua y Sub Proyectos, tanto por el Gobierno Territorial Rama y Kriol como por la Comisión Nacional de Desarrollo del Gran Canal de Nicaragua, esta adquiere carácter de Ley y debe ser publicada en La Gaceta, Diario Oficial de las y los Nicaragüenses.

Clausula Cuarta: De los Compromisos Conjuntos del Convenio de Consentimiento Previo, Libre e Informado

9. Para la implementación del presente Convenio de Consentimiento Previo, Libre e Informado, el Gobierno Territorial Rama y Kriol y la Comisión Nacional de Desarrollo del Gran Canal de Nicaragua adquieren los siguientes compromisos:

9.1 El Proyecto del Gran Canal Interoceánico de Nicaragua y Sub Proyectos se desarrollará obedeciendo las normas nacionales e internacionales de protección y sostenibilidad ambiental.

9.2 La Comisión Nacional de Desarrollo del Gran Canal Interoceánico de Nicaragua realizará una presentación oficial de los Estudios de Impacto Ambiental ante la Miembros del Gobierno Territorial Rama y Kriol.

9.3 Se desarrollarán acciones para contribuir a la mitigación y adaptación al cambio climático en el Territorio Rama y Kriol.

9.4 El Gobierno Territorial Rama y Kriol tendrá plena participación en las acciones de reforestación y desarrollo productivo en el Área Requerida.

9.5 Se garantizará la reforestación y protección de la Reserva Biológica Indio - Maíz.

9.6 Se diseñará e implementará un programa de formación y capacitación de jóvenes Rama y Kriol para acceder a empleos directos en el Proyecto del Gran Canal Interoceánico de Nicaragua y Sub Proyectos.

9.7 La protección, restauración y promoción del patrimonio cultural tangible e intangible del pueblo Indígena Rama y Afro descendiente Kriol será una prioridad del

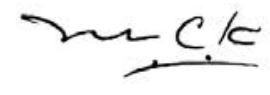


Proyecto de Desarrollo del Gran Canal de Nicaragua y Sub Proyectos.

9.8 Se desarrollarán acciones conjuntas para contribuir a mejorar las condiciones de seguridad comunitaria en el Territorio Rama y Kriol.

9.9 Se desarrollarán acciones conjuntas de cara a la implementación efectiva de la Ley del Régimen de Propiedad Comunal de los Pueblos Indígenas y Comunidades Étnicas de la Costa Caribe de Nicaragua (Ley 445).

Clausula Quinta: De la Planificación, Implementación y Monitoreo del Convenio de Consentimiento Previo, Libre $e$ Informado

10. Para la implementación del presente Convenio de Consentimiento Previo, Libre e Informado, el Gobierno Territorial Rama y Kriol y la Comisión Nacional de Desarrollo del Gran Canal de Nicaragua, conformarán un Comité de Planificación, Implementación y Monitoreo del Convenio.

Clausula Sexta: Duración del Convenio de Consentimiento Previo, Libre e Informado

11. El presente Convenio de Consentimiento Previo, Libre e Informado será de duración indefinida.

Clausula Séptima: De Adendas y Modificaciones al Convenio de Consentimiento, Previo, Libre e Informado

12. El presente Convenio de Consentimiento Previo, Libre e Informado para la Implementación del proyecto de Desarrollo del Gran Canal Interoceánico de Nicaragua, podrá ser modificado al término de 20 años luego de su firma y por el expreso consentimiento de ambas partes.

Clausula Octava: Del Registro del Convenio de Consentimiento, Previo, Libre e Informado

13. El presente Convenio de Consentimiento Previo, Libre e Informado para la Implementación del proyecto de Desarrollo del Gran Canal Interoceánico de Nicaragua,

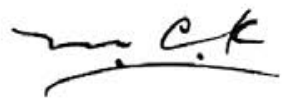


deberá ser debidamente notariado y registrado en el término de dos meses en el Registro Público de la Propiedad Inmueble y Mercantil en la ciudad de Bluefields, Región Autónoma de la Costa Caribe Sur, Nicaragua.

14. El presente Convenio de Consentimiento Previo, Libre e Informado para la Implementación del Proyecto de Desarrollo del Gran Canal Interoceánico de Nicaragua será entregado ante Naciones Unidas para su debido registro.

Dado en la Ciudad de Managua, Nicaragua a los 3 días del mes de mayo, del año 2016

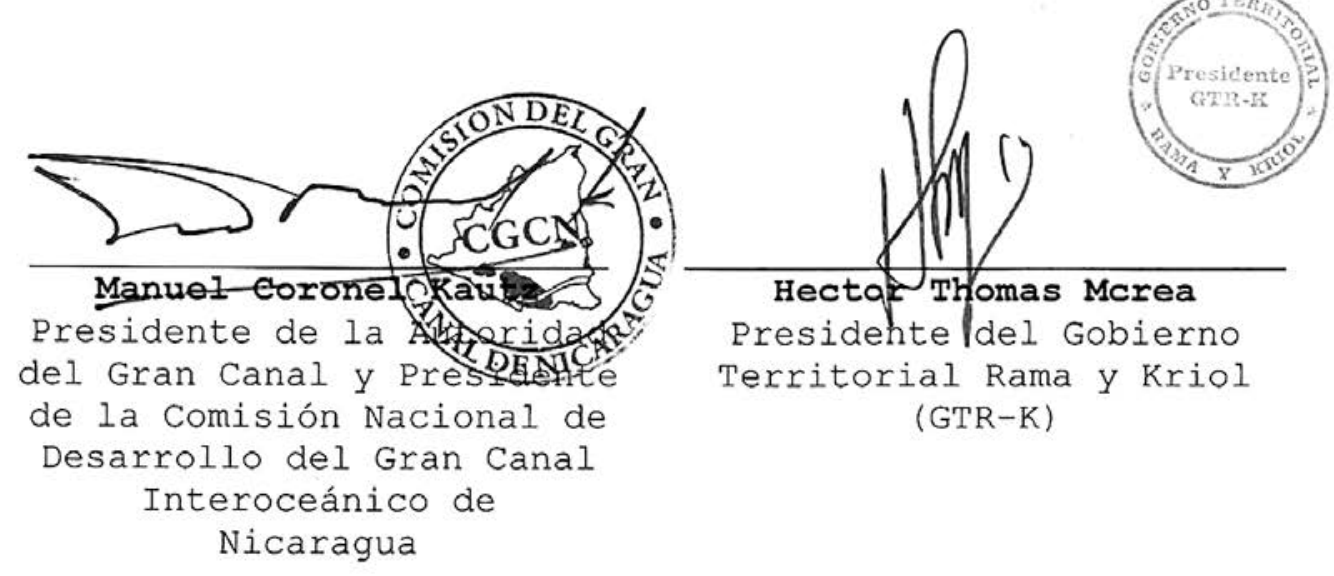

